\title{
EL CASO ALMACENES PARÍS, ALGUNAS CONSIDERACIONES EN TORNO AL CONCEPTO DE OFERTA PÚBLICA DE VALORES. \\ Comentario a oficio de Superintendencia de Valores y Seguros, 3 de marzo de $2005^{*}$
}

\author{
José Miguel Ried Undurraga ${ }^{* *}$
}

\begin{abstract}
RESUMEN: Al resolver administrativamente el caso "Almacenes París", la Superintendencia de Valores y Seguros amplió el concepto de oferta pública de valores, permitiendo por primera vez, para ciertos casos, ofertas públicas de valores que no están inscritos, pero que están en proceso de inscripción. Esto contribuye a flexibilizar las transacciones y a perfeccionar el mercado de valores.
\end{abstract}

ABSTRACT: The Securities and Insurance Superintendency has resolved the "Almacenes Paris case" widening the concept of securities public offer. For the first time, and only in certain cases, it has allowed public offers of non-registered securities which registration has been filed. This contributes to the transactions' flexibility and to perfect the securities markets.

\section{EL CONCEPTO DE OFERTA PÚBLICA DE VALORES}

La regulación del mercado de valores Chile, contenida principalmente en la Ley 18.045 ("Ley de Mercado de Valores" o "LMV"), consagra plena libertad para hacer ofertas de valores, en la medida que dichas ofertas sean hechas en forma privada.

La oferta realizada en forma pública queda reservada para los valores que hayan sido inscritos en el registro de valores, que lleva la Superintendencia de Valores y Seguros (la "SVS"). Esta regla implica que no se puede hacer ofertas públicas, incluyendo ofertas bursátiles, de aquellos valores que no estén debidamente registrados.

\footnotetext{
* Jurisprudencia comentada: "Solicitud de Consorcio Financiero S.A. en contra de Cencosud S.A.". Solicitud de retiro de una OPA en conformidad con lo dispuesto en el Artículo 214 de la Ley 18.045.

Materia: El concepto de oferta pública de valores y la situación que al respecto tienen los valores no inscritos pero que están en proceso de inscripción en el Registro de Valores.

Disposiciones Aplicables: Artículos $4^{\circ}$ y $6^{\circ}$ de la Ley No 18.045, sobre Mercado de Valores.

Doctrina: Es posible realizar ciertos actos que están prohibidos respecto de valores no inscritos, cuando se trata de valores que están en proceso de inscripción en el Registro de Valores de la Superintendencia de Valores y Seguros, pero cuyo registro aún está pendiente. La nueva postura de la SVS permite modernizar y flexibilizar las normas sobre oferta pública aplicables a valores que están en proceso de inscripción, incorporando, por ejemplo, la posibilidad de realizar actos preparatorios a la colocación usando la información que haya sido proporcionada a la SVS.

(El oficio de la Superintendencia de Valores y Seguros está publicado en su página internet, www.svs.cl)

${ }^{* *}$ Profesor de Derecho, Pontificia Universidad Católica de Chile.
} 
La idea de que los valores deben estar registrados en un ente regulatorio para permitir su oferta pública fue inspirada en las normas federales de valores de los Estados Unidos. Estas normas exigen, con algunas excepciones y peculiaridades, que los valores sean registrados ante la Securities and Exchange Commission para que puedan ser públicamente ofrecidos. Dicha obligación fue establecida en la Federal Securites Act de 1933. Con el sistema de registro de acciones y preparación del correspondiente prospecto se buscó que "hubiera una entrega de información total y equitativa en relación a distribuciones públicas de valores" 1 ya que el prospecto "está diseñado para entregar toda la información relevante necesaria para que los inversionistas puedan evaluar completamente las razones de fondo de su adquisición de valores; el prospecto es el vehículo que ubica a los inversionistas en el nivel más cercano posible al de los emisores y agentes colocadores, con la esperanza de que su compra no será una inversión sin valor o sobrevalorada" ${ }^{2}$.

Sin embargo, al aplicar estos principios, el legislador chileno definió lo que constituía oferta pública en una de las disposiciones más difusas y difíciles de interpretar de la Ley de Mercado de Valores.

El artículo $4^{\circ}$ de la referida ley señala:

"Se entiende por oferta pública de valores la dirigida al público en general o a ciertos sectores o grupos específicos de este".

Por su parte, el artículo $6^{\circ}$ señala:

"Solo podrá hacerse oferta pública de valores cuando estos y su emisor, hayan sido inscritos en el Registro de Valores" 3 .

Finalmente, el artículo 60, al establecer tipos penales relacionados con infracciones a la LMV, consagra penas de presidio para "los que hicieren oferta pública de valores sin cumplir con los requisitos de inscripción en el Registro de Valores que exige esta ley o lo hicieren respecto de valores cuya inscripción hubiere sido suspendida o cancelada".

\section{LA OFERTA PÚBLICA DE VALORES EN LA ERA DE INTERNET}

Analizando el concepto que da la LMV, no es difícil determinar cuándo una oferta ha sido dirigida al público en general y, por lo tanto, constituye una oferta pública. Por ejemplo, es fácil presumir que los anuncios que ofrecen la compra de ciertos valores publicados en medios de comunicación masivos, o en avisos fijados en la vía pública están dirigidos al público en general.

\footnotetext{
${ }^{1}$ CoX y LangeVOort (1997) pp. 4 y 5.

2 Cox y LangeVoort (1997) pp. 4 y 5.

3 En conformidad con el Artículo 183 de la LMV, también es posible hacer oferta pública de valores extranjeros en la medida que estén inscritos en el "Registro de Valores Extranjeros", registro que también está a cargo de la SVS.
} 
El problema está en las ofertas -siguiendo la redacción de la LMV- dirigidas a ciertos sectores o grupos específicos del público en general.

Por ejemplo, si yo soy accionista de una sociedad anónima cerrada junto con otros tres socios y les ofrezco comprar sus acciones, he hecho una oferta dirigida a los accionistas de mi sociedad. ¿Constituyen ellos un grupo específico del público en general? Aparentemente la respuesta es afirmativa. Sin embargo, este tipo de ofertas se hacen diariamente y nadie, incluyendo a la SVS, las consideraría como ofertas públicas de valores.

Lo que en realidad buscó el legislador al exigir la inscripción de los valores de oferta pública, al igual que en Estados Unidos, fue dar más información a personas que, por su escaso poder de negociación como inversionistas menores, no tenían acceso a la misma información que podían tener los actores importantes del mercado.

Otro de los problemas que presenta la definición es la dificultad que significa el poder determinar si una oferta de valores está dirigida o no a ciertas personas, sobre todo al tomar en consideración medios de comunicación que no existían en 1981, cuando se publicó la LMV, y que hoy son ampliamente utilizados.

Por ejemplo, ¿puede considerarse como una oferta dirigida al público en general chileno la página internet de un corredor de bolsa de Nueva York en la que ofrece valores no inscritos en Chile? Pongamos como ejemplo las páginas de las corredoras "etrade.com" o "schwab.com". La respuesta es que no, pues estas páginas no están dirigidas especialmente al público chileno.

Imaginemos ahora que etrade publica una página internet llamada etrade.cl, es decir, con la extensión "punto cl", que a su vez remita a la página etrade.com, donde se ofrecen las acciones. ¿Estamos en presencia de una oferta pública de valores no inscritos dirigida al público chileno en general? Recordemos que la página "punto cl" no ofrece nada y solo reenvía a la página "punto com".

Otra vuelta de tuerca: Si etrade publicara un aviso en un diario chileno con la frase "Visite etrade.com", ¿estaría haciendo oferta pública de valores? El aviso, claramente dirigido al público en general, no ofrece nada, solo invita a visitar una página en la que se ofrecen valores en forma general.

Pueden darse muchos supuestos como estos y las respuestas son cada vez más difíciles debido a la oscura redacción de la definición legal de oferta pública.

Sin perjuicio de lo anterior, el objetivo principal de este trabajo apunta a explorar otro aspecto de las ofertas públicas de valores.

\section{OPORTUNIDAD PARA HACER OFERTAS PÚBLICAS DE VALORES}

Según lo expuesto, solo es posible hacer oferta pública de valores que hayan sido inscritos en el registro de valores. Sin embargo, ¿cuál es la situación de los valores que no han sido aun inscritos, pero que están en proceso de inscripción?

\subsection{LA SITUACIÓN EN ESTADOS UNIDOS}

En Estados Unidos, como ya lo dijimos, la regla general es que no se puede hacer oferta pública de valores que no hayan sido "registrados" en la Securities and Exchange 
Commission (SEC). Sin embargo, la Securities Act de 1933 también señala que una vez que se ha iniciado el proceso de inscripción, aunque el registro no haya sido declarado aun efectivo, es posible realizar ciertas actividades que implican ofrecer de algún modo los valores.

En conformidad con lo estipulado en la Securities Act de 1933 y a la interpretación que de ella ha hecho la SEC, se ha entendido que los valores pueden encontrarse en tres situaciones respecto a su estado de inscripción ${ }^{4}$ :

\section{a) Período Previo}

Los valores cuyo registro aún no ha sido presentado ante la SEC para su aprobación y, en general, todos aquellos valores que no han sido inscritos, no pueden ser ofrecidos públicamente para su venta. Respecto de valores que se pretende registrar para ofrecerlos públicamente (por ejemplo, para abrirlos a la bolsa), esta restricción incluye que los valores no pueden ser vendidos ni su venta puede ser ofrecida, los agentes colocadores no pueden comprarlos ni ofrecer comprarlos. También queda prohibido el envío de cualquier tipo de información al mercado que pueda ser considerada que está condicionando al mercado para una posible oferta, lo que puede incluir en ciertos casos, por ejemplo, un aumento en la publicidad de los productos del eventual emisor.

Sin perjuicio de lo anterior, la futura emisión de valores no tiene que ser necesariamente un secreto. El emisor puede hacer algunos comunicados, como el hecho que planea colocar ciertos valores, el tipo y cantidad de valores que planea emitir y la forma y motivos que tendrá la oferta. Sin embargo, para que no sea una oferta de valores, no puede revelar el precio al que serán ofrecidos ni la identidad del agente colocador o underwriter.

\section{b) Periodo de Espera}

Esta etapa, también conocida como waiting period comienza cuando se presenta la solicitud de registro ante la SEC y termina con el registro de los valores. En este período intermedio los valores no pueden ser ofrecidos públicamente pues no han sido registrados aún. Sin embargo, es posible iniciar los trámites anteriores a la oferta. El emisor puede celebrar el contrato con el o los agentes colocadores, que en muchos casos implica una venta futura o promesa de venta de los valores. El agente colocador, por su parte, puede negociar con los otros corredores o agentes de valores que participarán en la colocación y contratar con ellos.

Las ofertas verbales (que no sean por radio o televisión) se permiten, pero no pueden celebrarse los contratos de compraventa. Es en este período en el que habitualmente se hacen las visitas y presentaciones a los inversionistas institucionales para promocionar los valores (road show). Sin embargo, no pueden distribuirse documentos o cualquier material escrito que contenga información sobre los valores, a excepción del prospecto provisorio que se presentó a la SEC para el registro. Este prospecto provisorio,

\footnotetext{
${ }^{4}$ Ver Guerrero (1995) pp. 389-415; y Cox, Hillman y LangeVoort (1997).
} 
también conocido como red herring, debe llevar en su portada una leyenda en color rojo que indica que el registro de los valores ha sido solicitado a la SEC, pero que no es válido; que el prospecto es provisorio y no constituye una oferta pública de los valores, que los valores no pueden venderse y que sus ofertas de compra no pueden aceptarse hasta que el registro sea declarado efectivo.

\section{c) Periodo Posterior al Registro}

Este período comienza cuando la SEC declara que el registro de los valores es efectivo. En este período pueden celebrarse los contratos de compraventa de los valores y hacer toda clase de ofertas, con la prevención de que las ofertas por escrito deben ir acompañadas de un ejemplar del prospecto de registro.

\subsection{LA SITUACIÓN EN CHILE}

Una interpretación estricta -y tal vez la más lógica- de la disposición del artículo $6^{\circ}$ de la LMV ("Solo puede hacerse oferta pública de valores cuando estos y su emisor, hayan sido inscritos en el Registro de Valores"), nos lleva a concluir la oferta pública de valores es solo aceptable una vez que la SVS ha concluido el trámite de su registro. En consecuencia, durante el "período de espera", comprendido desde el ingreso de la solicitud de registro a la SVS hasta el registro efectivo, no sería posible hacer ningún tipo de actividad que pudiera importar una oferta pública.

Lo anterior es relevante pues implicaría que en el "período de espera" no solo no se podría hacer una serie de actividades preparatorias para una colocación de valores, sino que tampoco podrían ofrecerse los valores que estuvieran en proceso de inscripción, en canje de valores inscritos, como en el caso de una OPA con canje de acciones.

Sabemos que la OPA es un proceso de oferta pública de adquisición de acciones, regulado por la LMV, a que deben someterse ciertas adquisiciones de acciones de sociedades anónimas abiertas en las que se busca adquirir el control de una sociedad o comprar ciertos porcentajes de sus acciones.

El artículo 203 de la LMV expresamente autoriza a que el precio ofrecido en una OPA de ciertos valores consista, a su vez, en "valores de oferta pública". Sin embargo, puede darse el caso que los valores que ofrece entregar en pago el oferente, no estén inscritos en el registro de valores al momento de hacer la OPA. En este escenario, ¿es posible que, junto con hacer la OPA, el oferente solicite el registro de los valores que usará para pagar el precio de los valores que ofrece comprar? ¿Se estaría vulnerando el artículo 203 al ofrecer pagar con valores que no son "de oferta pública" al momento de formularse la OPA? Y, más importante aun, ¿se puede considerar que los valores que están en proceso de inscripción están en una categoría distinta a la de los valores simplemente no inscritos, permitiéndose algunas excepciones a la regla de la oferta pública en el período de espera?

Estas preguntas han sido de algún modo resueltas por la Superintendencia de Valores y Seguros, al resolver administrativamente la solicitud que hiciera Consorcio Financiero S.A. de suspender la OPA formulada por Cencosud S.A. para tomar el control de Almacenes París S.A., según se verá a continuación. 


\subsection{El CASO PARÍs}

Para explicar el caso París con todas sus aristas sería necesario un trabajo más extenso que no pretendo hacer por ahora, por lo que solo haré una breve reseña basada principalmente en las presentaciones que las partes hicieron en su oportunidad ante la SVS.

A fines del año 2004 algunos miembros de la familia Gálmez, dueña mayoritaria de Almacenes París S.A. ("París"), vendieron su participación accionaria en dicha sociedad al grupo Consorcio Financiero ("Consorcio"). No vendió sus acciones don Jorge Gálmez, quien suscribió un "Convenio de cesión de acciones" con los compradores, por el cual se establecían derechos preferentes de compra y venta.

El 13 de enero de 2005 Cencosud S.A. (“Cencosud”) e Inversiones Mehuín, sociedad controlada por don Jorge Gálmez y dueña a esa época del 27\% de París, suscribieron un Acuerdo Marco, en el que se reguló la intención de Cencosud de comprar París, que tenía sus acciones inscritas en el registro de valores, y constituir un grupo controlador con Mehuín. Cencosud se obligaba a hacer una OPA por París. Mehuín se obligaba a concurrir a la OPA en la medida que los otros miembros del convenio de cesión de acciones lo autorizaran. Si Mehuín no hubiera podido participar en la OPA por sus obligaciones derivadas del convenio de cesión de acciones (como de hecho ocurrió), quedaba obligado por un pacto de accionistas con Cencosud y se obligaba a votar favorablemente una eventual fusión de Cencosud y París.

El 16 de febrero de 2005 Cencosud formuló una OPA en virtud de la cual ofreció comprar la totalidad de las acciones emitidas por París, las que se pagarían mediante un canje de acciones Cencosud, emitidas para ese efecto, a una relación de 1,1871 acciones París por cada acción Cencosud. Las acciones emitidas por Cencosud no estaban inscritas a esa fecha en el Registro de Comercio por lo que paralelamente, se solicitó su inscripción en el registro de valores.

El 24 de febrero de 2005, Consorcio Financiero S.A. hizo una presentación ante la SVS solicitando la suspensión y el retiro de la OPA de Cencosud, alegando, entre muchos otros argumentos, que a) Cencosud estaba haciendo una oferta pública de sus acciones al ofrecerlas como pago de la OPA, siendo que dichas acciones no estaban inscritas; y b) que en el marco de una OPA está permitido ofrecer solo valores inscritos como forma de pago del precio.

En sus presentaciones ante la SVS de fechas 24 de febrero y 2 de marzo de 2005, Consorcio señaló:

"Cencosud al ofrecer pagar a los destinatarios de la OPA las acciones que estos decidan ofrecerle con acciones Cencosud, está, en el hecho, haciendo oferta pública de estas. Considerando que las acciones Cencosud no están inscritas en el Registro, Cencosud está infringiendo las normas citadas en el párrafo anterior y desconociendo las facultades de la Superintendencia para reparar, observar o rechazar el registro de las referidas acciones de Cencosud.

En efecto, la OPA tendría la calidad de medio u oferta de adquisición de acciones de París y forma y oferta de colocación de acciones de nueva emisión de Cencosud, todo ello públicamente. 
Cabe hacer presente que las disposiciones de los artículos 4, 6 y 60 de la LMV no distinguen entre ofertas condicionadas, sujetas a otras modalidades o puras y simples, sino que simplemente prohiben y penalizan, respectivamente, la oferta pública de valores no inscritos en el Registro".

"En consecuencia la OPA comporta a un tiempo la calidad de oferta de adquisición de acciones París y de oferta pública de colocación de acciones Cencosud. Lo anterior implica una abierta infracción a lo dispuesto en el Artículo $\sigma^{\circ}$ de la Ley $N^{\circ} 18.045$ ("LMV") que solo permite hacer oferta pública de valores cuando estos hayan sido inscritos en dicho Registro".

Sin embargo, luego de recibir los descargos de Cencosud, la SVS rechazó la solicitud de Consorcio mediante oficio ordinario No 2207 de fecha 3 de marzo de 2005.

La SVS consideró que Cencosud no estaba haciendo una oferta pública de sus valores sino que solo estaba haciendo una oferta de compra de las acciones emitidas por París, condicionada a que las acciones de Cencosud estuvieran inscritas al momento del canje. Respecto a que los valores que se ofrecen en canje en el marco de una OPA deban estar inscritos, la SVS hizo ver que la Norma de Carácter General número $104^{5}$ expresamente autoriza a que en una OPA se puedan ofrecer valores que aun no han sido emitidos como pago del precio.

Al respecto, el numeral 11 de la Sección II de dicha norma señala que, dentro de la información que debe proporcionar el que formula la OPA, debe indicar la forma en que financiará la oferta y "si se tratare de una oferta de canje de valores, deberá detallarse la forma en que el oferente ha adquirido, adquirirá o emitirá tales valores" (el subrayado es mío). Queda claro que pueden ofrecerse valores que ni siquiera han sido emitidos como forma de pagar los que se ofrecen comprar en una OPA.

El oficio de la SVS señala textualmente: "[...] cuando el medio de pago consiste en valores, ello no constituye oferta pública de los mismos y por lo mismo, se requiere que se encuentren inscritos en el Registro de Valores al momento de efectuarse la entrega o pago correspondiente. Si lo anterior no fuere suficiente, debe entenderse que a través de dicha norma [la Norma de Carácter General número 104] esta Superintendencia ejerce su facultad aclarativa e interpretativa contemplada en el artículo $4^{\circ}$ de la Ley $N^{\circ} 18.045$, en esta materia" 6.

Aunque la SVS opine lo contrario, estimo que en el caso que comentamos Cencosud hizo una verdadera oferta pública de sus acciones, no una oferta de venta, sino que de canje, lo que en la práctica tiene el mismo efecto. Como ya vimos, el artículo 4 de la LMV no exige que la oferta deba ser de compra o de venta, para ser considerada como "pública", sino que simplemente señala que "se entiende por oferta

\footnotetext{
${ }^{5}$ La Norma de Carácter General número 104 es una regulación de la SVS en la que se establecen normas sobre el procedimiento para realizar una OPA.

${ }^{6}$ El citado artículo $4^{\circ}$ dispone: "La Superintendencia podrá eximir ciertas ofertas públicas del cumplimiento de alguno de los requisitos de la presente ley, mediante resoluciones de carácter general”. Las facultades de la SVS para interpretar la ley se encuentran en realidad en el artículo $4^{\circ}$ del D.L. 3.538 de 1980, que crea la Superintendencia de Valores y Seguros.
} 
pública de valores la dirigida al público en general o a ciertos sectores o grupos específicos de este". Es decir, no excluye del concepto de oferta pública una oferta de canjear valores.

$\mathrm{Al}$ ofrecer dar en pago sus acciones no inscritas, Cencosud estaba en la práctica ofreciéndolas en forma pública. Afirmar lo contrario implicaría sostener que el canje de acciones París por acciones Cencosud no implicaba canjear acciones Cencosud por acciones París, siendo que evidentemente son dos caras de la misma moneda.

Cencosud ofreció un canje de valores en su OPA, figura contemplada en nuestro Código Civil bajo el nombre de permuta o cambio. El artículo 1900 del Código Civil equipara los efectos de la permuta a los de la compraventa al señalar: "Las disposiciones relativas a la compraventa se aplicarán a la permutación en todo lo que no se oponga a la naturaleza de este contrato; cada permutante será considerado como vendedor de la cosa que da, y el justo precio de ella a la fecha del contrato se mirará como el precio que paga por lo que recibe en cambio".

Por lo tanto, la SVS aceptó un tipo de oferta pública de valores que no estaban inscritos, pero que estaban en proceso de inscripción. En los hechos, la SVS consideró que existía una situación especial para aquellos valores que están en proceso de inscripción, similar a lo que existe en la regulación de valores de Estados Unidos.

Entre los argumentos que dio la SVS en su oficio para rechazar la solicitud de Consorcio, incluyó expresamente que los valores estaban en proceso de inscripción:

"A mayor abundamiento, la sociedad emisora se encuentra inscrita en el Registro de Valores de esta Superintendencia asi como sus acciones emitidas con anterioridad al aumento de capital acordado en la junta de accionistas celebradas el 31 de enero del año en curso. A su vez, las acciones de pago que se acordaron emitir en la referida junta, y que en sí, constituyen el medio de pago señalado, se encuentran en proceso de inscripción ante esta Superintendencia. [...]

De este modo, el pago respectivo mediante acciones de Cencosud S.A. sería efectuado una vez que dichas acciones ya se encuentren inscritas en el Registro correspondiente, y de no ser así, la Oferta Pública de Adquisición de Acciones sobre Empresas Almacenes París S.A. caducaría ipso facto, y por ende, la OPA no podría declararse exitosa." (El subrayado es mío).

Es decir, en ciertos casos, y sin que medie una excepción legal expresa al respecto, es posible entender que puede hacerse una oferta pública condicionada de valores no inscritos, en la medida que estén en proceso de inscripción. La condición consiste en que los valores se inscriban efectivamente. De no ocurrir este hecho, la transacción no se podría llevar a efecto.

\section{CONCLUSIONES}

Nuestra legislación solo permite las ofertas públicas de valores si estos están inscritos en el registro de valores de la SVS. Por ello resulta relevante determinar con alguna 
precisión no solo la extensión del concepto "oferta pública", sino que desde cuándo pueden realizarse las ofertas y sus actos preparatorios en casos de valores que están en proceso de inscripción.

El concepto de oferta pública que da la Ley de Mercado de Valores es difuso, sus límites son difíciles de precisar, lo que produce una incertidumbre jurídica sobre la materia, especialmente al considerar medios de comunicación como internet que permiten ofrecer valores en formas tales que el concepto de oferta pública, establecido en nuestra legislación hace más de 20 años, queda superado.

Adicionalmente, para efectos de determinar si puede hacerse oferta pública de valores, nuestra regulación no ha considerado la diferencia que existe entre un valor no inscrito y un valor que está en proceso de inscripción. Esta diferenciación, recogida en legislaciones como la de Estados Unidos, flexibiliza la forma de hacer transacciones de valores, lo que redunda en una mayor celeridad y eficiencia para realizarlas, colaborando a que los mercados de valores sean más perfectos.

Sin perjuicio de lo anterior, en el caso Almacenes París, la SVS ha dado una interpretación novedosa a la Ley de Mercado de Valores y ha considerando que los valores que están en proceso de inscripción tienen efectivamente un estatus diferente respecto de aquellos valores que simplemente no están inscritos.

En el caso París en concreto, estimó que era posible ofrecer públicamente valores no inscritos, pero que estuvieran en proceso de inscripción, en canje o permuta por valores inscritos. La SVS basó esta interpretación en la Norma de Carácter General No 104, emitida por esa misma superintendencia, pero además, y así lo dice textualmente el oficio, en que estaba ejerciendo su "facultad aclarativa e interpretativa contemplada en el artículo $4^{\circ}$ de la Ley $N^{o} 18.045$, en esta materia", lo que da especial fuerza a esta resolución y la hace aplicable en forma general.

Con el caso que se comenta, se ha abierto una puerta para otorgar un estatus diferente a los valores que están en proceso de inscripción, permitiéndose que a su respecto puedan realizarse ciertos actos que por lo general están prohibidos para valores no inscritos.

La nueva postura permite presumir que la SVS podrá, por la vía de interpretar la legislación de valores, modernizar y flexibilizar aun más las normas sobre oferta pública aplicables a valores que están en proceso de inscripción, incorporando, por ejemplo, la posibilidad de realizar actos preparatorios a la colocación usando la información que haya sido proporcionada a la SVS, en forma similar a lo dispuesto por las normas federales de valores de Estados Unidos. 


\section{BIBLIOGRAFÍA CITADA}

Cox, James D., Hillman Robert W. y Langevoort, Donald C., (1997): Securities Regulation: cases and materials (New York, Aspen Law \& Business) 1.266 pp.

Guerrero V., Roberto (1995): "Sumario de aspectos legales sobre la colocación de Adra en los mercados internacionales", Revista Chilena de Derecho, vol. 22, No 3: pp. 389-415.

\section{NORMAS CITADAS}

Ley 18.045, de Mercado de Valores. Diario Oficial. 22 de octubre de 1981.

Norma de Carácter General No 104. Superintendencia de Valores y Seguros, 2001. 